\title{
Trichoblastic Fibroma of the Skin Mimicking Primary or Secondary Breast Cancer
}

Prvulović Bunović Nataša ${ }^{1,2}$, Boban Jasmina ${ }^{1,2}$, Šveljo Olivera ${ }^{2,3}$, Djilas Dragana ${ }^{1,2}$, Mihailović Jasna ${ }^{1,4}$ and Nikin Zoran $^{1,5}$

1. Faculty of Medicine, University of Novi Sad, Novi Sad 21000, Serbia

2.Diagnostic Imaging Center, Oncology Institute of Vojvodina, Sremska Kamenica 21204, Serbia

3.Faculty of Technical Science, University of Novi Sad, Novi Sad 21000, Serbia

4.Department of Nuclear Medicine, Oncology Institute of Vojvodina, Sremska Kamenica 21204, Serbia

5.Department of Pathology, Oncology Institute of Vojvodina, Sremska Kamenica 21204, Serbia

\begin{abstract}
We report a case of a 71-year- old female with trichoblastic fibroma, a rare benign tumor of the skin, found in the left breast, associated with an invasive contralateral breast cancer. On clinical examination, a solitary, firm nodule was found in the subcutaneous tissue of the left breast with no changes in the overlying skin. Radiological examination showed disconcordant results. Conventional mammography and ultrasound suggested benign nature, while magnetic resonance mammography and spectroscopy raised the presumption of the malignant nature of the lesion. After performing excisional biopsy, the diagnosis of trichoblastic fibroma was established. Microscopically, it was composed of fibrous stroma, basaloid germs and strands and lace-like epithelial components, with no obvious connection with overlying epithelium or adjacent adnexal structures.
\end{abstract}

Key words: Trichoblastic fibroma, differential diagnosis, breast, MR (magnetic-resonance) mammography, ${ }^{1} \mathrm{H}$ MR spectroscopy.

\section{Introduction}

Adnexal skin tumors are a heterogeneous group of rare tumors, especially arising from skin overlying the breast parenchyma. This type of tumor sometimes can be difficult to diagnose without histology, because of wide variety of clinical presentations [1-4].Cases of apocrine poroma, syringomatous adenoma of the nipple, cylindroma, hidradenoma, syringocystadenoma papilliferum, pilomatrixoma have been reported $[1,2$, 4-11]. To the best of our knowledge, only one case of trichoblastic tumor in the skin of the breast has been reported up to now [12].

The role of MR (magnetic-resonance) imaging in diagnosis of breast pathology has increased over the past decade. Breast MRM (MR mammography) is the most sensitive modality for invasive breast cancer

Corresponding author: Prvulović Bunović Nataša, M.D., Ph.D., associate professor, research fields: breast imaging and oncologic imaging. detection, but it has limited specificity usually leading to unnecesary biopsies. A study by Liberman et al. [13] showed that MR imaging detected cancer that was not visible on physical examination and mammography in the contralateral breast in $5 \%$ of women recently diagnosed with breast cancer. MRM increases detection of mammographically or ultrasonographically occult lesions in ipsilateral or contralateral breast, but the actual clinical impact of these incidental cancers is unknown [14]. Modern MRM protocols include pharmacokinetic modeling, DWI (diffusion-weighted imaging) and MRS (MR spectroscopy). MRS can be detected with choline that is precursor of the phospholipids composing cell membranes. Increase in choline peak reflects increased membrane metabolism and/or degradation, thus enabling differentiation between benign and malignant lesions and serving as an indicator of tumor activity and viability [15-17]. Recent data supports the role of 
multiparametric breast imaging since it provides more accurate analysis than any of the imaging modalities alone.

We report a case of a woman with a trichoblastic fibroma in the skin of the left breast and a primary breast carcinoma contralaterally, in which clinical and radiological differential diagnosis of bilateral breast pathology was rather difficult.

\section{Case Report}

A 71-year-old female from a rural part of the country was referred for consultation due to a lesion in her right breast that had been present for two years. She noticed the infiltration of the skin a year before. Upon physical examination, our attention was brought to a subcutaneously localized, round, 2-cm palpable mass at the junction of upper quadrants in the contralateral breast. The mass in the left breast was painless and with no visible changes in the overlying skin. In addition, the woman reported that both lesions had occurred at the approximately same time. The personal and family history of breast cancer was unremarkable. A presumptive clinical and radiological diagnosis of either primary or secondary breast cancer was established for the left breast.

\section{(1) Radiological findings}

The patient was immediately referred to mammography and bilateral breast ultrasound. Mammography revealed an oval, well-demarcated mass with gently lobulated margins at the junction of upper quadrants of the left breast, adjacent to the skin (Fig. 1a). According to the ACR (American College of Radiology) BI-RADS (Breast Imaging Reporting and Data System) classification, the lesion was assigned BI-RADS 3 category [18]. Additional ultrasound examination showed a well-circumscribed solid mass lesion with complex echo pattern and with posterior acoustic features, also designated as BI-RADS 3 lesion (Fig. 1b).

At the time, in our center a large study on multi-voxel proton MR spectroscopy ( $\left.{ }^{1} \mathrm{H}-\mathrm{MRS}\right)$ of breast lesions was being conducted and the patient signed a fully-informed written consent to take part in

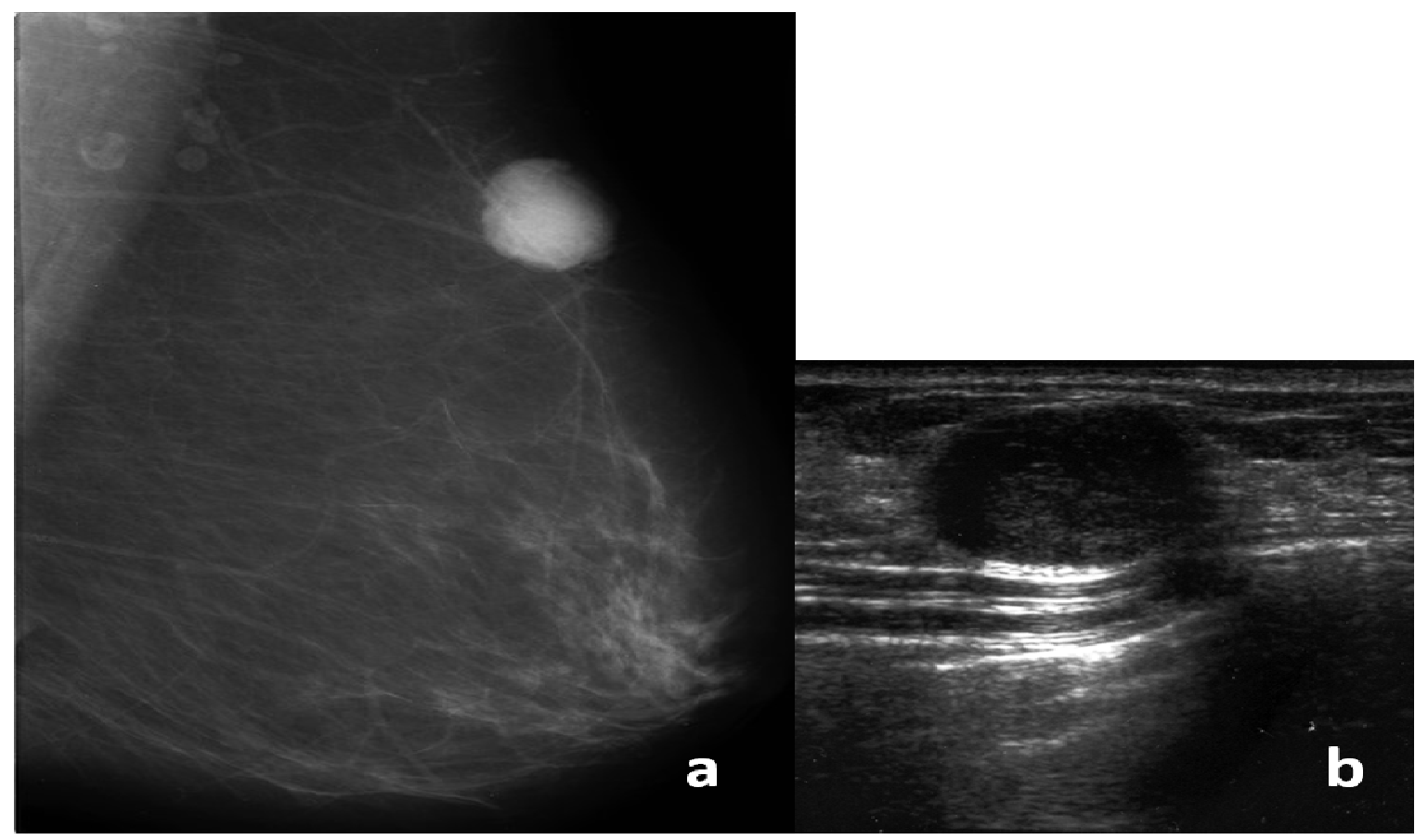

Fig. 1. Mediolateral oblique mammogram of the left breast showing a well-demarcated mass with gently lobulated margins in the upper quadrant (a); Ultrasound image shows a circumscribed solid mass lesion with complex echo pattern and posterior acoustic features (b). 
this study. Firstly, a standard bilateral contrast-enhanced MRM was performed at Avanto 1.5T MR unit (Siemens, Erlangen, Germany), using a standard 3D FLASH dynamic post-contrast protocol with a dedicated breast coil. At MRM, the lesion in the left breast presented as an oval, well-demarcated solid mass with smooth margins (Fig. 2a). Post-contrast dynamic study revealed a heterogeneous and strong early enhancement followed by a plateau time course (Type II curve) (Fig. 2b). This helped classifying the tumor as BI-RADS 4. Afterwards, 3D ${ }^{1} \mathrm{H}$ spectroscopic imaging (MRSI) of metabolites with voxel grid of $4 \times 4 \times 4 \mathrm{~cm}$ and voxel size of $1 \mathrm{~cm}^{3}$ was obtained in order to differentiate the nature of the lesion (malignant vs. benign) according to the presence of choline in the spectra. The mass showed elevated peak of choline (Cho) at $3.2 \mathrm{ppm}$, which suggested malignant nature of lesion (Fig. 2c). An excisional biopsy was performed and benign cutaneous lesion was confirmed on histology, without any need for additional procedures.
In the right breast, however, clinical examination and all radiological findings implied a BI-RADS 5 lesion (Fig. 2d). The patient underwent right mastectomy and histopathological diagnosis of the invasive lobular carcinoma was established. After mastectomy, the patient underwent adjuvant radiotherapy and chemotherapy with anthracyclines and taxanes.

(2) Histopathological findings of left breast tumor

The excised specimen of left breast mass was a well-circumscribed lesion in the dermis and subcutis, measuring $23 \times 21 \mathrm{~mm}$. Considering the gross features, the lesion was slightly lobulated, firm and yellowish-grayish in color. Microscopically, it consisted of fibrous stroma, basaloid germs, basaloid strands and lace-like epithelial components (Fig. 3a). There was no contact of the basaloid component with the epidermis (Fig. 3b). A final diagnosis of a trichoblastic fibroma was established.
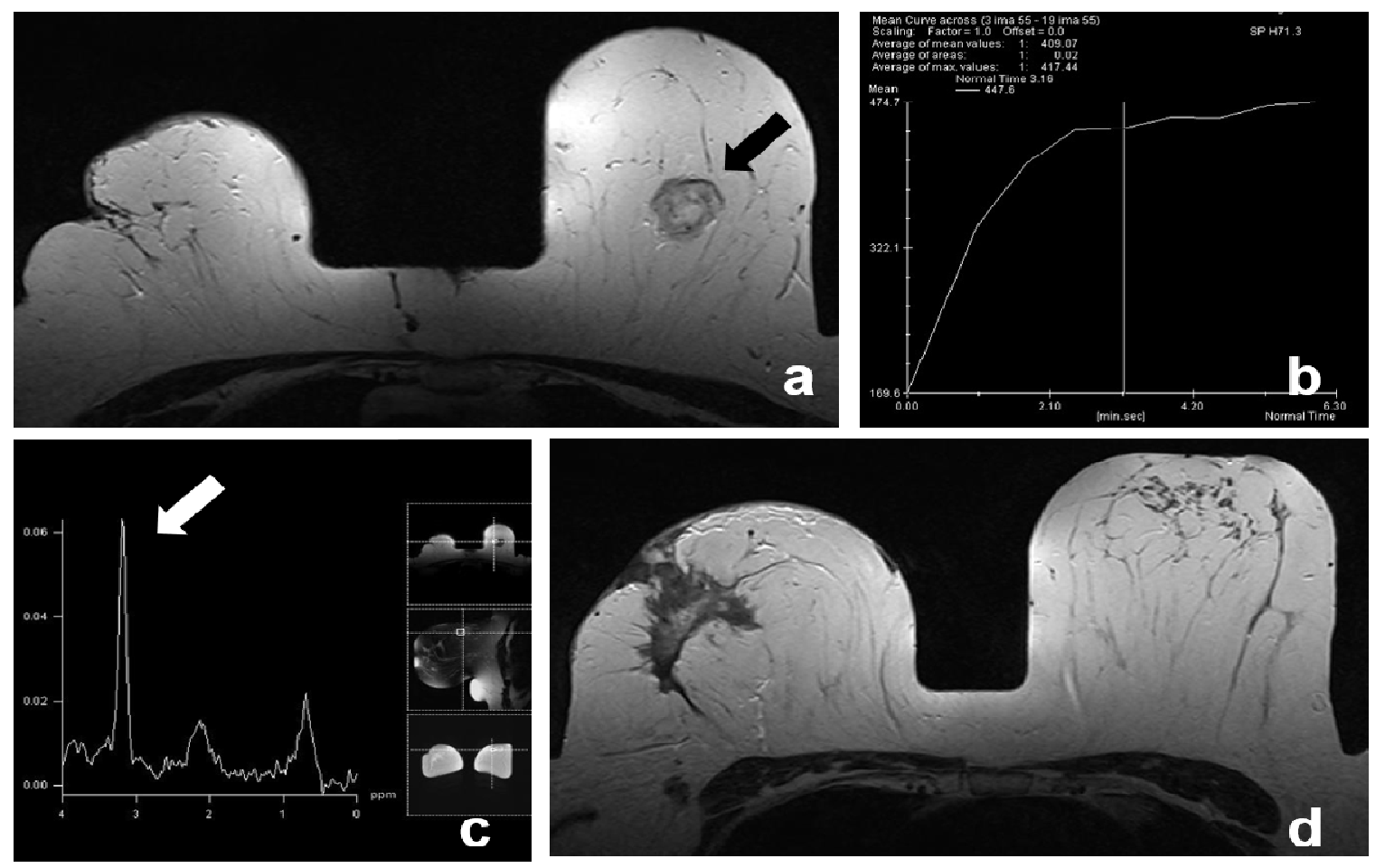

Fig. 2. AxialT2-weighted image of the left breast showing a well-demarcated solid mass with smooth margins and heterogeneous internal structure (a); after contrast administration lesion exhibits a plateau time course (Type II curve) (b); multi-voxel MR spectroscopy shows a Cho peak at $3.2 \mathrm{ppm}$, indicative of malignancy (c); axial bilateral T1-weighted post-contrast image shows an extensive vascularized invasive carcinoma in the right breast (d). 

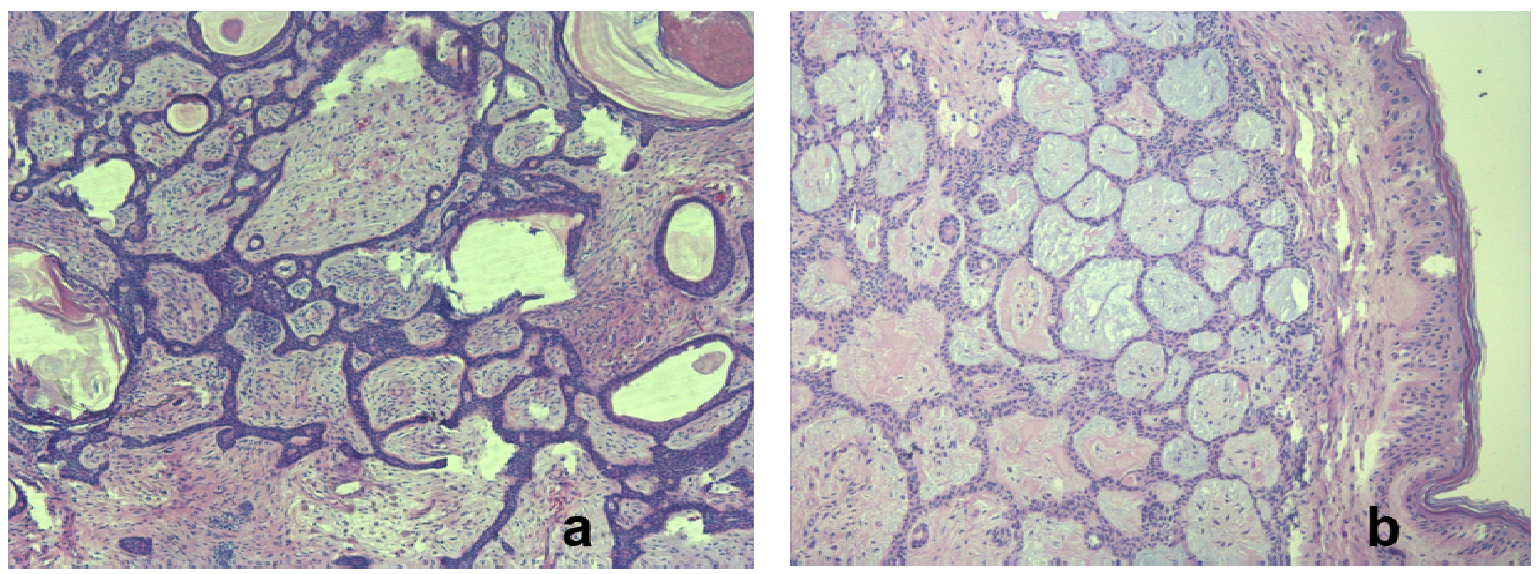

Fig. 3. Photomicrograph demonstrating the presence of fibrous stroma, basaloid germs and strands as well as lace-like epithelial components (a), with no connection to the epidermis (b).

\section{Discussion}

The main purpose of this case report is to draw the attention to skin adnexal tumors that may be considered in differential diagnosis of primary or secondary tumors of the breast. Trichoblastic fibroma is an uncommon trichogenic tumor with partial to complete mesenchymal induction toward follicular differentiation. Classical clinical presentation of this tumor is a large solitary dermal or subcutaneous nodule measuring $1-8 \mathrm{~cm}$, variable in localization, with a slight predominance in the face and pelvic girdle region [19]. Trichoblastic fibroma is, according to Headington, one of three neoplasms implied by term "trichoblastic tumors", with various degrees of follicular differentiation and stromal component [20]. Many authors have described subtle variations within the spectrum of follicular germinative neoplasms, utilizing terms such as trichoblastoma, trichoblastic fibroma, and trichogenic trichoblastoma [21]. All neoplasms hidden behind the term of trichoblastic tumors share common architectural attributes of a benign neoplasm: relative symmetry, sharp circumscription, and no or insignificant cytological atypia. In addition, all trichoblastic tumors are linked by a predominance of basaloid (follicular germinative) cells with enveloping fibrocytic stroma that varies in degree [22].A predominance of follicular germinative cells within basaloid germs may exist; they can be arrayed as either small or large nodules, with a hint of an intervening fibrous stroma, and these lesions are called "trichoblastoma".

In this case we presented, the presumptive clinical diagnosis, based on the physical and standard radiological examinations (mammography, ultrasound), implied a benign lesion. However, MRM and ${ }^{1} \mathrm{H}$ MRS, which were performed as a part of a large clinical trial, suggested the malignant nature of the lesion (BI-RADS 4). Namely, postcontrast kinetics raised the level of suspected malignancy, with a heterogeneous, early strong enhancement. In addition, an elevated Cho peak presented on the multi-voxel MR spectroscopy, was indicative of the malignant nature of the described lesion in the breast. Taken into account a large co-existent invasive carcinoma in the contralateral breast, differential diagnosis of a primary or secondary breast tumor was included. Metastatic origin could not be excluded since the mass was solitary, well circumscribed, superficially located and of clinically benign appearance.

To the best of our knowledge, this is the first MR imaging and $3 \mathrm{D}{ }^{1} \mathrm{H}$ spectroscopic imaging performed in a trichoblastic fibroma in general. MRS is a relatively novel diagnostic modality useful for obtaining information regarding the metabolic activity in the breast. It has found its place in defining biochemical profiles mainly in the field of brain pathology, while in breast pathology it is still in 
development. MR spectroscopy has been suggested as an adjunct to the breast MR imaging to distinguish benign from malignant breast lesions [23,24].Elevated Cho compounds are thought to be a marker of an active tumor in the breast with reported sensitivity of $70-100 \%$ and specificity of $67-100 \%[25,26]$.

Reviewing the literature, we have not found any previous reports on the in vivo MRS findings of trichoblastic fibroma. Although trichoblastic fibroma represents a benign skin tumor, it presented an elevated Cho peak characteristic for malignant lesions. The aberrant choline metabolism is established as universal metabolic indicator of cancer. However, MRS of the breast is a diagnostic modality still in progress, indicating that quantification of choline compounds may lead to increased reliability in differentiation of breast tumors [27-29].

Namely in our patient, the presence of choline, regardless of concentration, was proven to be misleading, probably due to mesenchymal origin of the tumor [25]. Radiological diagnosis of the breast lesion should take into account both morphologic and dynamic features of the mass, and lead to a comprehensive and clinically useful conclusion. However, when considering a breast lesion, one should bear in mind that, although rarely, in breast tissues other than glandular benignant and malignant tumors can also be found.

\section{Conclusions}

In conclusion, further improvement of MRM and MRS in breast pathology is needed to increase the specificity of these imaging modalities. On the other hand, research on the molecular basis of the association between the skin changes and internal cancer, as well as the recognition of these cutaneous signs and symptoms independent of malignancy should eventually help the clinician to establish a correct diagnosis [30,31].

\section{References}

[1] Pujani, M., Madaan, G., Jairajpuri, Z., Jetley, S., Hassan,
M., and Khan, S. 2016. "Adnexal Tumors of Skin: An Experience at a Tertiary Care Center at Delhi.” Annals of Medical and Health Sciences Research 6 (5): 280-5.

[2] Azma, A., Tawfik, O., and Casparian, J. M. 2001. “Apocrine Poroma of the Breast.” Breast J. 7: 195-8.

[3] Cappello, Z. J., Kasdan, M. L., Augenstein, A. C., and Shaheen, S. P.2013. "Squamous Cell Carcinoma in an Epidermoid Cyst.” Eplasty 13: ic44.

[4] Hamilton, A., Young, G. I., and Davis, R. I. 1987. "Pilomatrixoma Mimicking Breast Carcinoma."Br. J. Dermatol 116: 585-6.

[5] Toyoshima, O., Kanou, M., Kintaka, N., Miyashita, M., Shigematsu, S., and Sano, J. 1998. "Syringomatous Adenoma of the Nipple: Report of a Case.” Surg. Today 28:1196.

[6] Gokaslan, S. T., Carlile, B., Dudak, M., and Albores-Saavedra, J. 2001. "Solitary Cylindroma (Dermal Analog Tumor) of the Breast: A Previously Undescribed Neoplasm at This Site.” Am. J. Surg. Pathol 25: 823-6.

[7] Nonaka, D., Rosai, J., Spagnolo, D., Fiaccavento, S., and Bisceglia, M. 2004. "Cylindroma of the Breast of Skin Adnexal Type: A Study of 4 Cases.” Am. J. Surg. Pathol 28: 1070-5.

[8] Kazakov, D. V., Vanecek, T., Belousova, I. E., Mukensnabl, P., Kollertova, D., and Michal, M. 2007. "Skintype Hidradenoma of the Breast Parenchyma with $\mathrm{t}$ (11; 19) Translocation: Hidradenoma of the Breast.” Am. J. Dermatopathol 29: 457-61.

[9] Nowak, M., Pathan, A., Fatteh, S., Fatteh, S., and Lopez, J. 1998. "Syringocystadenoma Papilliferum of the Male Breast.” Am. J. Dermatopathol 20: 422-4.

[10] Imperiale, A., Calabrese, M., Monetti, F., and Zandrino, F. 2001. "Calcified Pilomatrixoma of the Breast: Mammographic and Sonographic Findings.” Eur. Radiol 11: 2465-7.

[11] Viero, R. M., Tani, E., and Skoog, L. 1999. "Fine Needle Aspiration (FNA) Cytology of Pilomatrixoma: Report of 14 Cases and Review of the Literature.” Cytopathol 10: 263-9.

[12] Shimazaki, H., Anzai, M., Aida, S., Endo, H., Kato, K., Yamasaki, T., et al. 2001. "Trichoblastoma of the Skin Occurring in the Breast: A Case Report.”Acta. Cytol. 45: 435-40.

[13] Liberman, L., Morris, E. A., Kim, C. M., Kaplan, J. B., Abramson, A. F., Menell, J. H., et al. 2003. “MR Imaging Findings in the Contralateral Breast of Women with Recently Diagnosed Breast Cancer.” Am. J. Roentgenol 180 (2): 333-41.

[14] Gupta, D., and Billadello, L.2017. "Breast MR Imaging in Newly Diagnosed Breast Cancer.” Radiol Clin. North Am. 55 (3): 541-52. 
[15] Funke, M. 2016. "Diagnostic Imaging of Breast Cancer: An Update.” Radiologie 56 (10): 921-38.

[16] Bidlek, M., Kovacs, E., Feher, K., and Godeny, M. 2015. "New Trends and Novel Possibilities in the Diagnostic Imaging of Breast Cancer.” Magy. Onkol. 59 (1): 44-55.

[17] Katz-Brull, R., Seger, D., Rivenson-Segal, D., et al. 2002. "Synthesis Metabolism and Reduced Choline-Ether-Phospholipid Synthesis.” Cancer Res. 62: 1966-70.

[18] American College of Radiology (ACR) Breast Imaging Reporting and Data System Atlas (BI-RADS Atlas) 5th Edition.Reston, Va. American College of Radiology. 2013.

[19] Altman, D. A., Mikhail, G. R., Johnson, T. M., and Lowe, L. 1995. "Trichoblastic Fibroma. A Series of 10 Cases with Report of a New Plaque Variant.” Arch. Dermatol 131 (2): 198-201.

[20] Headington, J. T. 1990. "Tumors of Hair Follicle Differentiation.”In: Pathology of the Skin. Farmer, E., Hood, A., Eds. New York: McGraw-Hill, 596-614.

[21] Ackerman, A. B., Reddy, V. B., Soyer, H. P. 2001. Neoplasms with Follicular Differentiation. 2nd Ed. New York: Ardor Scribendi, 1109.

[22] Bettencourt, M. S., Prieto, V. G., and Shea, C. R. 1999. "Trichoepithelioma: A 19-YearClinicopathologic Re-evaluation.” J. Cutan Pathol 26 (8): 398-404.

[23] Montemezzi, S., Cavedon, C., Camera, L., et al. 2017. " $1 \mathrm{H}-\mathrm{MR}$ Spectroscopy of Suspicious Breast Mass Lesions at 3T: A Clinical Experience.”Radiol Med.122: 61.

[24] Djilas-Ivanovic, D., $\quad$ Prvulovic, $\quad$ N.,
Bogdanovic-Stojanovic, D., Vicko, F., Sveljo, O., and Ivkovic-Kapicl, T. 2009. "Dermatofibrosarcoma Protuberans of the Breast: Mammographic, Ultrasound, MRI and MRS Features.” Arch Gynecol Obstet 280 (5): 827-30.

[25] Tse, G. M., Yeung, D. K., King, A. D., Cheung, H. S., and Yang, W. T. 2007. "In vivo Proton Magnetic Resonance Spectroscopy of Breast Lesions: An Update.” Breast Cancer Res. Treat 104 (3): 249-55.

[26] Baltzer, P. A.,and Dietzel, M. 2013. "Breast Lesions: Diagnosis by Using Proton MR Spectroscopy at $1.5 \mathrm{~T}$ and 3.0 T-Systematic Reviews and Meta-Analysis.”Radiology 267: 735-46.

[27] Begley, J. K. P., Redpath, T. W., Bolan, P. J., and Gilbert, F. J. 2012. "In vivoProton Magnetic Spectroscopy of Breast Cancer: A Review of the Literature."Breast Cancer Research 14: 207.

[28] Baik, H-M, Yu, H. J., Chen, J-H., Nalcioglu, O., and Su, M. Y. 2008. "Quantitative Correlation between ${ }^{1} \mathrm{H}$ MR Spectroscopy and Dynamic Contrast-Enhanced MRI of Human Breast Cancer.” Magn.Reson Imaging 26 (4): 523-31.

[29] Cheng, M., Bhujwalla, Z. M., and Glunde, K. 2016. “Targeting Phospholipid Metabolism in Cancer." Front Oncol 6: 266.

[30] Thiers, B. H., Sahn, R. E., and Callen, J. P. 2009. “Cutaneous Manifestations of Internal Malignancy." CA Cancer J. Clin .59 (2): 73-98.

[31] Wienbeck, S., Herzog, A., Kinner, S., and Surov, A. 2016. "Magnetic Resonance Imaging Findings of Intramammary Metastases.” Clinical Imaging 40 (3): 361-4. 